\title{
Magnetic Resonance Imaging in a Patient with Pacemaker
}

\author{
Jaime Giovany Arnez Maldonado, Maria Euda Pereira, Karina Rabelo de Albuquerque, \\ Jorge Pires \\ Manaus, AM - Brazil
}

The patient is a 24-year-old female with a dual-chamber pacemaker, who had intracranial hypertension, progressive visual loss, and several inconclusive cranial tomographies. She underwent magnetic resonance imaging, even though that diagnostic method is absolutely contraindicated in patients with pacemakers.

Magnetic resonance imaging (MRI) is a noninvasive diagnostic method that provides highly defined anatomic images and is widely used in neurology.

Magnetic resonance imaging is formally contraindicated in patients with pacemakers (PM). This means that patients wearing these prostheses, who have another pathology associated that requires $\mathrm{MRI}$, cannot undergo that examination because of the complications and risks.

We report the case of a patient with a pacemaker who underwent MRI.

\section{Case Report}

The patient is a 24 year-old female with a dual-chamber pacemaker DDDR (Guidant, Insígnia I Plus model) implanted 15 months earlier, due to repeated syncope caused by neurocardiogenic syndrome of the cardioinhibitory type. After implantation, the patient's symptoms significantly improved.

Seven months after craniocerebral trauma due to an accident, the patient evolved with neurologic findings of intracranial hypertension and progressive loss of the visual field. Then the patient underwent multiple cranial computed tomographies and lumboperitoneal shunt; however, no diagnostic conclusion was reached about the cause of the pathology. Cranial magnetic resonance imaging and angioresonance were requested by the neurosurgical team to define the diagnosis; these examinations, however, were contraindicated because the patient had a cardiac pacemaker. We chose to perform the procedures considering the risks and benefits.

The following protocol was followed for performing the examination: 1) The patient must know the risks of undergoing the exami-

Hospital Santa Júlia e Hospital Universitário Dona Francisca Mendes - Manaus

Mailing address: Jaime Giovany Arnez Maldonado - Rua "O", 133 -

Apto 702 - Parque 10 - Cep 69050-290 - Manaus, AM, Brazil

E-mail: jaimearnez@cardiol.br

Sent for publication: 07/08/2004

Accepted for publication: 10/06/04

English version by Stela Maris Costalonga nation; 2) Nonpacemaker-dependent patient; 3) Consent agreement signed by the patient and a family member; 4) Telemetric control of the pacemaker before and after MRI; 5) Medical team support comprising a cardiologist specialized in cardiac stimulation, an anesthesiologist, and a radiologist; 6) Complete material for reanimation; 7) Chest X-ray in PA view before and after MRI.

The equipment used was a magnetic resonance device at 0.5 Tesla, Contour model, manufactured by General Electric.

The procedure sequence was as follows:

Previous assessment through telemetry of the pacemaker generator, impedance of the electrodes, threshold and sensitivity control, bipolar programming of pacing and sensing, DDD mode of stimulation.

Venous puncture, cardiac monitoring, continuous pulse oximetry during the entire procedure.

The patient was exposed to the RMI device for approximately 50 minutes. Normally, 6 sequences are performed with a duration of 5 minutes. In the specific case of this patient, she asked to leave the MRI tunnel (chamber where the magnet generating the magnetic field and radiofrequency is located) at every sequence, which increased the total length of stay at the room.

During the first sequence, an irregular cardiac rhythm compatible with atrial fibrillation was observed. Every time the patient left the MRI tunnel, she was asked about symptoms. After the first sequence, the patient reported a "burning" sensation in the precordial region and at the site of the generator associated with vibration and palpitations. In the last sequences, the patient persisted with the precordial burning, which was then more intense, causing breathing difficulty associated with hoarseness. Sedation and venous corticoid therapy were performed to improve the symptoms and allow completion of the examination.

All hemodynamic parameters were kept stable during the entire procedure. At the end, the patient was transferred to the Intensive Care Unit, and her pacemaker was reassessed. After monitoring, sinus rhythm was observed.

The pacemaker was reassessed by use of telemetry, with no difficulties on interrogation. An increase was observed in the atrial command threshold from 0.4 volts with $0.4 \mathrm{~ms}$ to 0.7 volts with $0.4 \mathrm{~ms}$, and the electrodes remained with the same impedance. The atrial and ventricular sensitivities were not altered. The programming showed no alteration, and no damage was detected in the pacemaker circuit or in the generator's battery. The event counter function documented one atrial fibrillation (AF) (fig. 1) and one atrial tachycardia (AT) (fig. 2), which coincided with the 
patient's entrance into the MRI tunnel and activation of the radiofrequency field. The control chest X-ray showed no change in regard to the position of the electrodes and generator (fig. 3 ).

Regarding the result of the cranial images obtained through MRI, no type of artifact was generated by the presence of the pacemaker (fig. 4 and 5).

\section{Discussion}

Due to the risks of adverse effects of the magnet and the signal of radiofrequency generated by the MRI system on the pacemaker, MRI performance is currently considered absolutely contraindicated in patients with that prosthesis ${ }^{1-4}$. The potential adverse interactions between the pacemaker and MRI include the inhibition of pacemaker exit, pacemaker warming, pacemaker vibration, asynchronic stimulation of the pacemaker, induction of atrial fibrillation, induction of ventricular fibrillation, switch mode malfunction, rapid atrial stimulation, rapid ventricular stimulation, and alteration in the pacemaker programming with potential damage to the pacemaker circuit or system dislocation ${ }^{5-8}$.

MRI generates potent electromagnetic forces in the form of a static magnetic field and pulsatile radiofrequency magnetic field ${ }^{9}$. Malfunction of the PM results from interference with radiofrequency during MRI performance and may cause total inhibition in the atrial and ventricular exit, or, on the contrary, may cause dangerous

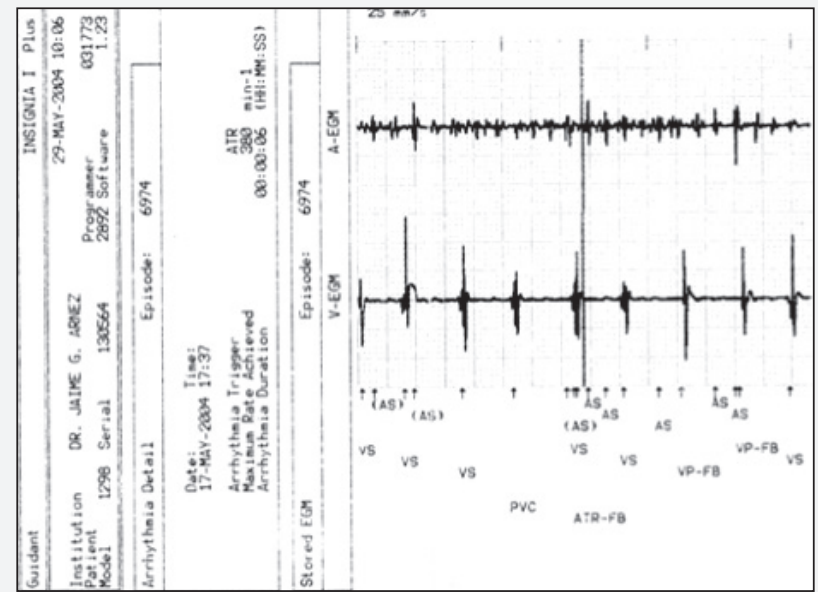

Fig. 1 - Atrial fibrillation (A-EGM: Atrial channel; V-EGM: Ventricular channel).

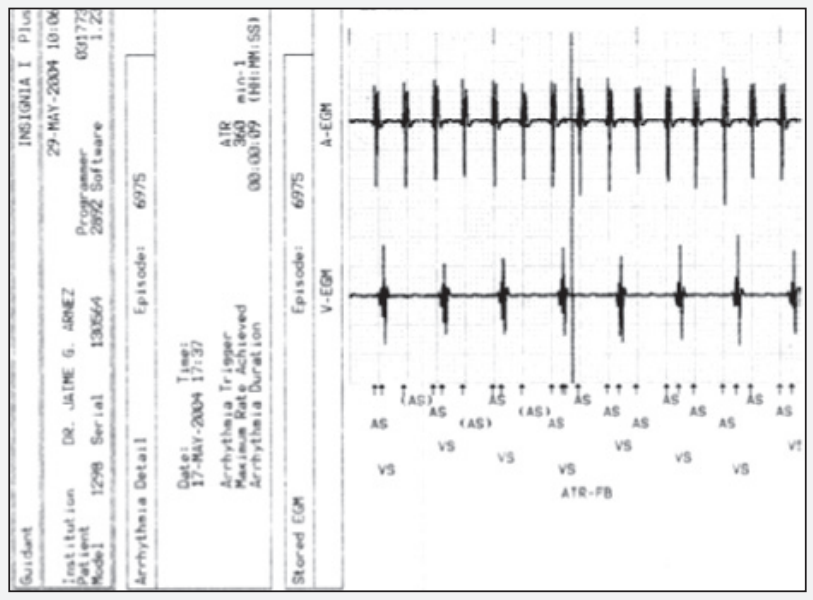

Fig. 2 - Atrial tachycardia (A-EGM: Atrial channel; V-EGM: Ventricular channel). stimulation at high frequencies in the chambers, leading to atrial or ventricular fibrillation and consequent deleterious effects ${ }^{3,5-7}$.

Another adverse effect caused by the radiofrequency energy is warming of the electrodes, which should be considered when patients are exposed to MRI, this being the factor that causes precordial discomfort during the examination and change in the PM command threshold ${ }^{1,6}$.

Therefore, MRI performance in patients with PM is formally contraindicated.

Safety and viability of MRI in patients with $\mathrm{PM}$ is an issue that has gained importance ${ }^{10}$. Some situations in clinical practice require the performance of MRI to provide valuable information. To deny

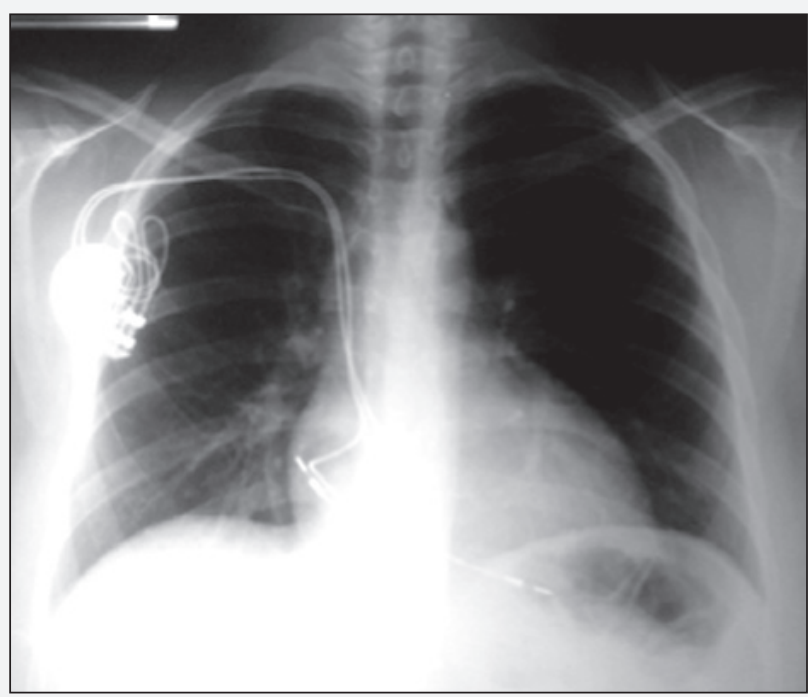

Fig. 3 - Chest X-ray after magnetic resonance imaging.

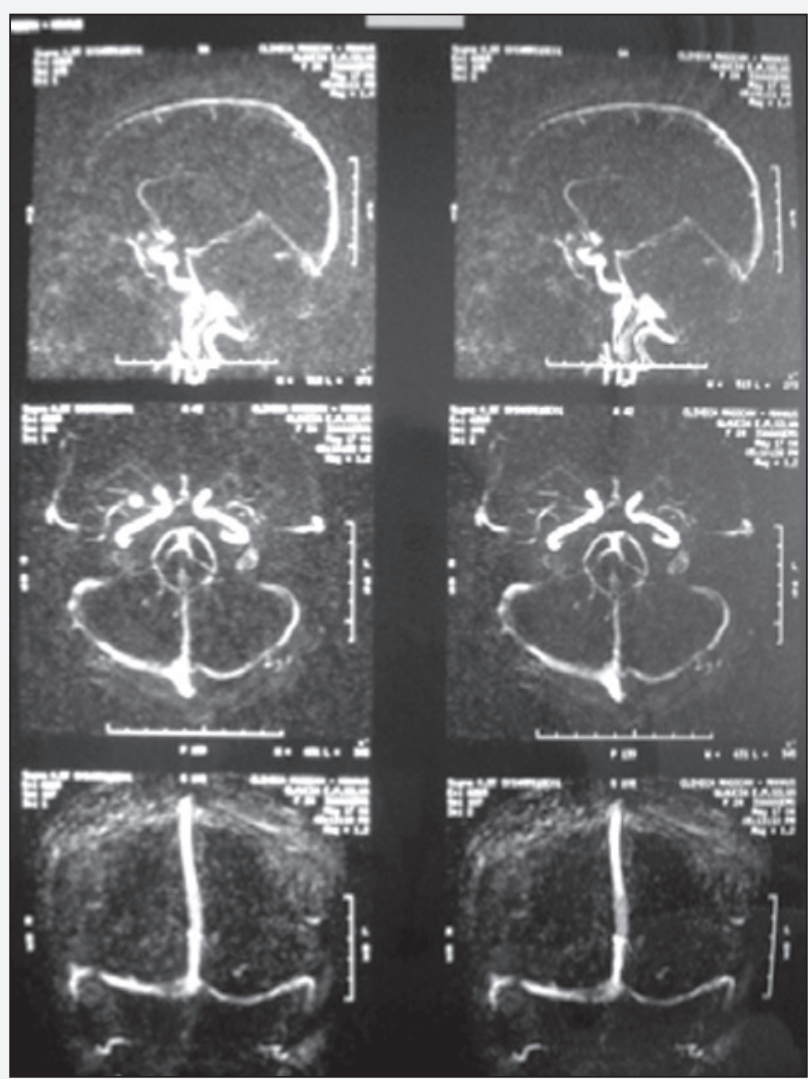

Fig. 4 - Cranial angioresonance. 


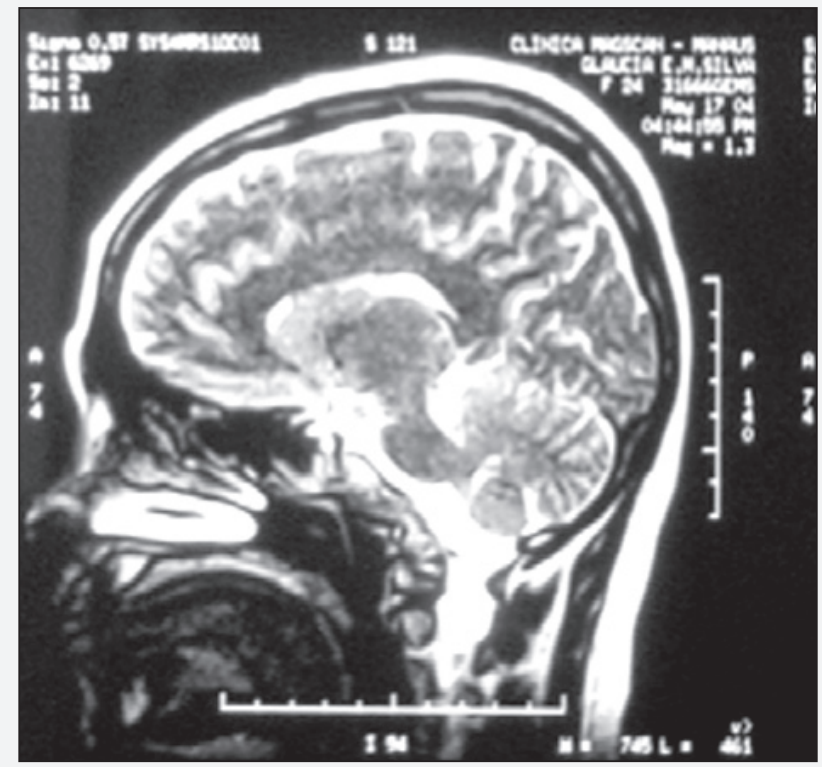

Fig. 5 - Cranial magnetic resonance.

the performance of that procedure to patients may have a significant impact on patient management.

The performance of MRI in nonpacemaker-dependent patients would eliminate potential complications, if the stimulation was inhibited during the examination ${ }^{1,11}$.
Edward T. Martin et $\mathrm{al}^{1}$ in a case series of 54 patients with pacemakers (nondependent) undergoing $62 \mathrm{MRI}$ examinations at 1.5T, assessing 61 pulse generators and 107 electrodes, showed a safety profile in that series of patients.

Vahlhaus et $\mathrm{al}^{10}$ carried out a prospective study with 32 patients undergoing $34 \mathrm{MRI}$ examinations at 0.5T. They assessed the stimulation and sensitivity thresholds, impedance of the electrode, the voltage, current, and impedance of the battery, and showed that MRI at 0.5T does not cause irreversible changes in the PM system.

Lauck et al $^{12}$ assessed the influence of MRI at $0.5 T$ on the new generation of PM, using ghost models and the following stimulation modes: VVI, VVIR, VOO, DDD, DDDR, and DOO. Influence was identified neither on the stimulation function nor on the programming of the PM. The function "event counter" remained intact.

In our patient, the precordial burning sensation, presence of supraventricular arrhythmia (AF/AT), and change in the PM command threshold may be attributed to warming and vibration of the electrode system caused by the radiofrequency energy emitted during image obtainment. In addition, no other alteration was observed in the pacemaker system.

In this non-PM-dependent patient, MRI at 0.5 T caused certain risks and complications (symptoms and arrhythmias), but proved to be potentially safe, with low and calculated risks. In conclusion, the absolute contraindication to performing MRI in patients with PM has been reassessed.

\section{References}

1. Martin ET, Coman JA, Shellock FG et al. Magnetic resonance imaging and cardiac pacemaker safety at 1.5-Tesla. JACC 2004;43: 1315-24.

2. Consenso SOCESP-SBC sobre Ressonância Magnética em Cardiologia. Arq Bras Cardiol 1995;65: 469-74.

3. Hayes DL, Holmes DR, Gray JE. Effect of 1.5T nuclear magnetic resonance imaging scanner on implanted permanent pacemakers. J Am Coll Cardiol 1987; 10:782-6.

4. Roguim A, Zviman MM, et al. Modern Pacemaker and Implantable Cardioverter/ Defibrillator Systems Can Be Magnetic Resonance Imaging Safe. Circulation 2004; 110: 475-82.

5. Erlebacher JA, Cahill PT, Pannizzo F et al. Effect of Magnetic Resonance imaging on DDD pacemakers. Am J Cardiol 1986; Feb 57: 437-40.

6. Achenbach S, Moshage W, Diem B et al. Effects of magnetic resonance imaging on cardiac pacemakers and electrodes. Am Heart J 1997; 134: 467-73.
7. Holmes DR, Hayes DL, Gray JE et al. The effects of magnetic resonance imaging on implantable pulse generators. Pacing Clin Electrophysiol 1986; 9: 360-70.

8. Hayes DL, Vliestra RE. Pacemaker malfunction. Ann Intern Med 1993; 119: 828-35.

9. Fontaine JM, Mohamed FB, Gottlieb C et al. Rapid ventricular pacing in a pacemaker patient undergoing magnetic resonance imaging. Pacing Clin Electrophysiol 1998;21:1336-9.

10. Vahlhaus C, Sommer T, Lewalter T et al. Interference with cardiac Pacemakers by magnetic resonance imaging: are there irreversible Changes at 0.5 tesla? Pacing Clin Electrophysiol 2001;24 (4Pt1);489-95.

11. Inbar S, Larson J, Burt T et al. Case report: nuclear magnetic resonance imaging in a patient with a pacemaker. Am J Med Sci 1993; 305:174-5.

12. Lauck G, von Smekal A, Wolke S et al. Effects of nuclear magnetic resonance imaging on cardiac pacemakers. Pacing Clin Electrophysiol 1995; 18: 1549-55. 